\title{
Condroblastomas del carpo: caso clínico y revisión de la literatura
}

\section{Carpal Chondroblastomas: Case Report and Review of the Literature}

\author{
Ángel Ferreres-Claramunt ${ }^{1}$ Leonardo Lázaro $^{2}$ \\ ${ }^{1}$ Cirugía de la Mano y Extremidad Superior, Institut Kaplan, Passeig \\ Bonanova, 9, 08022 Barcelona, España \\ 2 Departamento de Miembro Superior, Servicio de Ortopedia y \\ Traumatología, Clínica Francesa SRL/ Clínica de Mano S.A, \\ Address for correspondence Ángel Ferreres Claramunt, PhD, Cirugía \\ de la Mano y Extremidad Superior, Institut Kaplan, Passeig Bonanova, \\ 9, 08022 Barcelona, España \\ (e-mail: angelferreres@institut-kaplan.com).
} Guaymallén, Mendoza, Argentina

Rev Iberam Cir Mano

\section{Resumen \\ Palabras Clave \\ - condroblastomas \\ - huesos del carpo \\ - hueso escafoides \\ - tumor óseo benigno}

Los condroblastomas son tumores benignos poco frecuentes (su incidencia corresponde a menos del $1 \%$ de los tumores óseos primarios), de aparición habitual en la epífisis de huesos largos; siendo el fémur distal y la tibia proximal sus sitios de aparición más habituales. Es raro el asiento de esas lesiones en los huesos del carpo. A pesar de que sigue siendo considerado un tumor benigno, puede tener un comportamiento agresivo, tanto como recidiva local como produciendo metástasis. Presentamos un caso de un paciente de 16 años que presentó un condroblastoma de escafoides con recidivas en dos ocasiones tras un tratamiento convencional de legrado e injerto.

Chondroblastomas are rare benign tumors (less than $1 \%$ of primary bone tumors) that affect more frequently long bones epiphysis such as distal femur or proximal tibia. In spite of being considered a benign tumor, a more aggressive behavior, as local recurrence or metastasis, has been reported. We present a case of chondroblastoma that affected the scaphoid of a 16 years old boy, and that recurred twice after conventional treatment, till a more radical surgery was performed.

\section{Introducción}

El condroblastoma ( $\mathrm{CB}$ ) es un tumor de los que se conocen como formadores de cartílago catalogado por Jaffe y Lichtenstein $^{1}$ en 1942, localizado en la epífisis de huesos largos siendo más frecuente alrededor de la rodilla (fémur distal y tibia proximal), con un $30 \%$ de los casos publicados, seguido por el húmero proximal con un $20 \%$. Su aparición en huesos cortos del carpo y tarso es rara, ${ }^{2-5}$ más frecuente en calcáneo y en astrágalo. ${ }^{6}$ También se ha descrito de origen extraesquelético. ${ }^{7}$ Se presentan con mayor frecuencia en personas de sexo masculino (2:1) y mayormente durante la segunda década de la vida. ${ }^{2}$ Respecto a todos los tumores óseos, los que afectan al carpo constituyen alrededor del $0,15 \%$, de los cuales el 13,6\%, en largas series, son CB. Asientan con mayor frecuencia en el escafoides. ${ }^{2-4,8}$ Cabe mencionar aquí que en la tercera edición del libro sobre tumores óseos de 1978 de Dahlin, no venía reflejado ningún caso de CB que afectara a los huesos del carpo. ${ }^{9}$

El tratamiento más adecuado para ese tipo de lesiones es el legrado o resección intralesional y el relleno del defecto con injerto óseo autólogo. ${ }^{2,5,8,10}$ No obstante, se han descrito recidivas locales más o menos agresivas ${ }^{4,5,11-14}$ e incluso metástasis. $^{14-20}$ received

January 7, 2018

accepted

July 4, 2018
DOI https://doi.org/

10.1055/s-0038-1667321. ISSN $1698-8396$.
Copyright $\odot$ Thieme Revinter

Publicações Ltda, Rio de Janeiro, Brazil
License terms

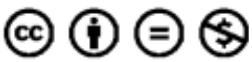




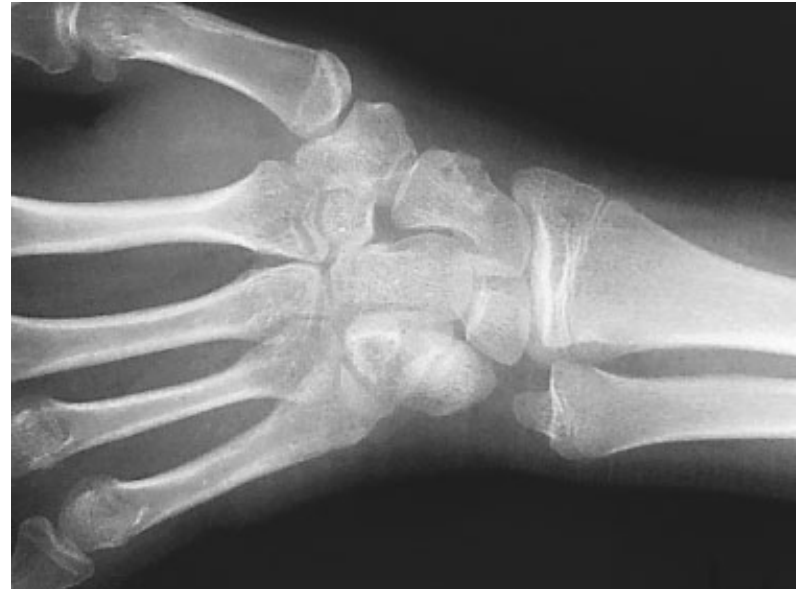

Fig. 1 Rx en la que se puede observar lesión en borde radial de escafoides.

\section{Caso Clínico}

Se trata de un paciente masculino de 16 años de edad que consultó por dolor, inflamación y limitación funcional en la muñeca izquierda sin antecedente traumático.

En el examen radiográfico se observó una imagen lítica en la zona radial del tercio medio del escafoides. Como estudios complementarios, se realizaron una TC y una RMN que fueron informadas de la presencia de una lesión lítica excéntrica e insuflante, situada en el tercio medio y polo distal del escafoides en su región radial que medía $8 \times 9 \mathrm{~mm}$. Dicha lesión tenía bordes esclerosos polilobulados con pequeñas áreas de discontinuidad de la cortical ósea con una impronta hacia partes blandas adyacentes. No existían signos de fractura patológica. Se observaban, calcificaciones nodulares intralesionales (-Figs. 1-3)
Se realizó una biopsia escisional y el estudio histopatológico informó de una lesión constituida por proliferación de células de núcleo redondo, cromatina epitelioide eosinófila, con áreas de matriz condral y zonas lobuladas, algunas células mostraban calcificaciones formando un patrón enrejado. En la periferia se observaban trabéculas óseas con signos de remodelación, pleomorfismo celular era leve y sin mitosis, siendo diagnosticado de condroblastoma.

El paciente presentó una buena evolución postoperatoria, sin complicaciones y sin dolor durante un año y medio. Veinte meses después de dicha cirugía consultó nuevamente por reaparición de dolor y edema en la muñeca (-Fig. 4). Se solicitaron estudios en los que pudo observarse la recidiva del tumor (-Fig. 5).

Se reintervino para realizar un legrado más amplio con fresado y aporte de un injerto estructural de la metáfisis radial por debilitamiento del escafoides en la segunda resección. (-Fig. 6)

Durante el seguimiento de esa última cirugía, el paciente evolucionó también de forma satisfactoria. En los estudios de control se observaba el injerto integrado (-Fig. 7) y estuvo asintomático hasta dos años después de la última cirugía en que reapareció el dolor. Nuevo TAC y RMN mostraron una mayor destrucción del escafoides y una afectación importante de tejidos blandos. (-Fig. 7).

En esa ocasión, se realizó una biopsia previa tomando, además, muestras de partes blandas y de hueso. El resultado puso de manifiesto una invasión de las partes blandas vecinas con cierto grado de celularidad y mayor componente cartilaginoso. Se interpretó como condrosarcoma de bajo grado. $^{21,22}$ Tras esa segunda recidiva se optó por la exéresis del escafoides y realizar una artrodesis semilunar-huesogrande $^{23}$ (-Fig. 8). Cinco años después de esa última

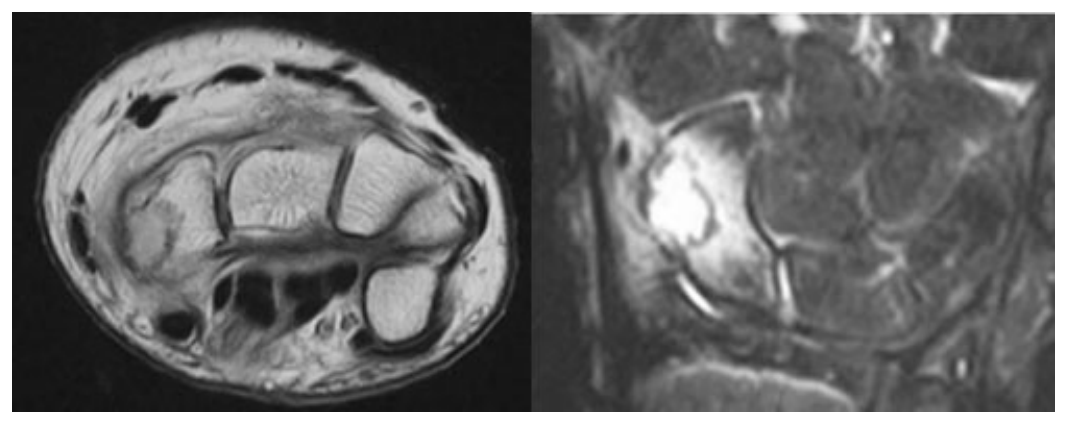

Fig. 2 RMN se observa la lesión insuflante del escafoides en ambos cortes.

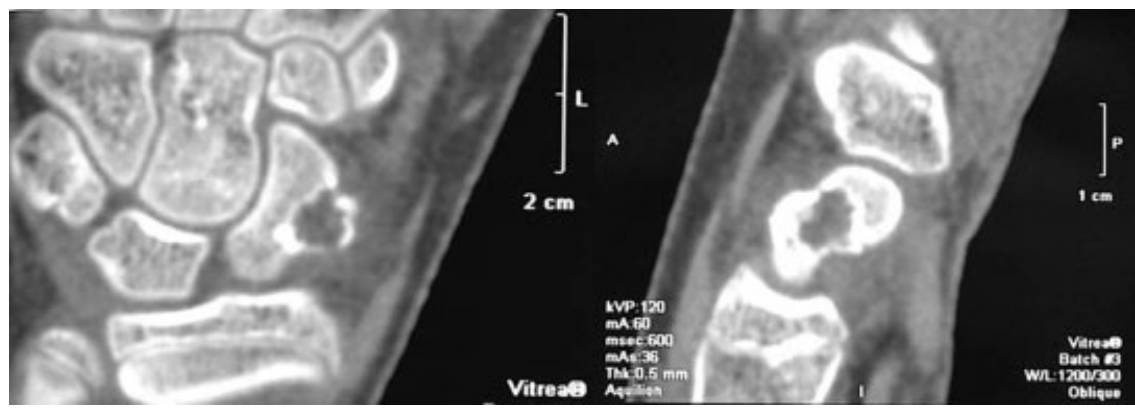

Fig. 3 TAC, se observa lesión con invasión del borde radial del escafoides. 


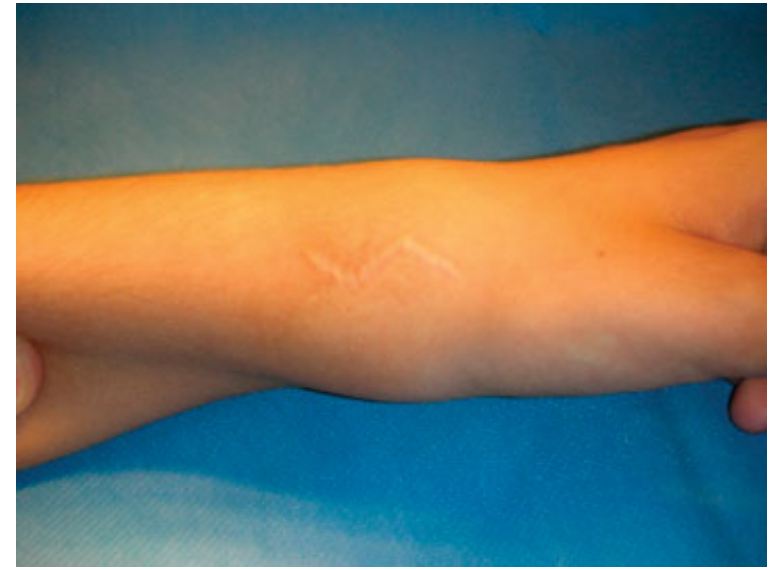

Fig. 4 Tumefacción sobre el aspecto radial de la muñeca.

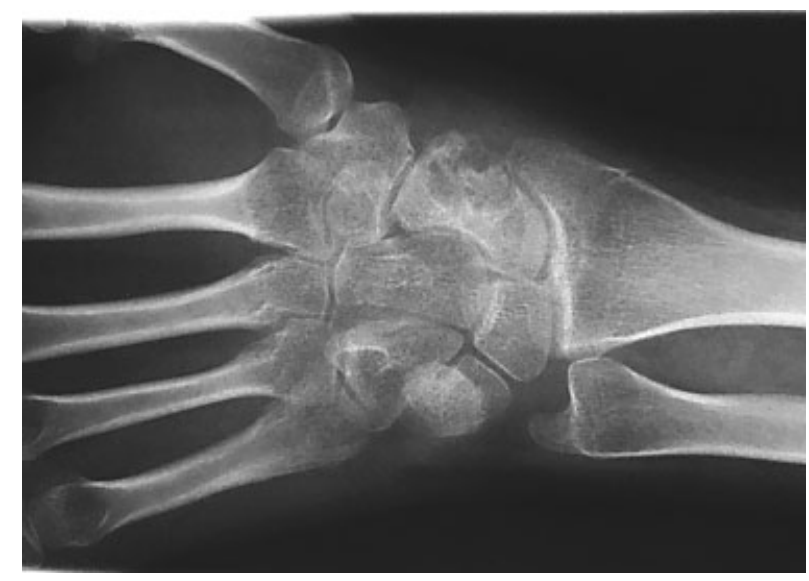

Fig. 5 Se observa la recidiva del tumor en el sitio original.

cirugía, el paciente no ha presentado recidivas y se encuentra asintomático.

\section{Discusión}

El Condroblastoma es un tumor localizado, benigno, con una buena respuesta al tratamiento habitual consistente en un legrado de la lesión y relleno con hueso esponjoso tanto en las localizaciones habituales, ${ }^{14,21}$ como en las carpianas, ${ }^{5,8,10} \mathrm{Sin}$

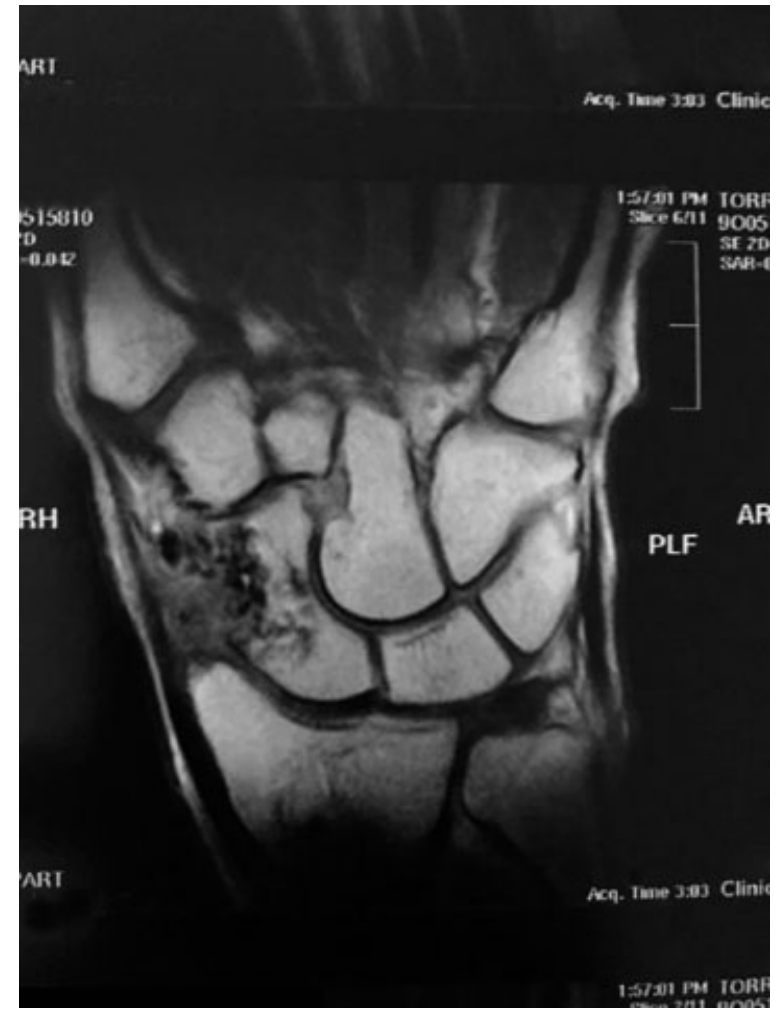

Fig. 7 Imagen de RMN en que se observa la destrucción del escafoides y la afectación de tejidos blandos.

embargo, a veces puede tener un comportamiento más agresivo $^{13,22,23}$ ocasionando recidivas en un $10 \%$ de casos e incluso metástasis en un 3,3\% según largas series publicadas. ${ }^{14}$ Más rara es la transformación maligna del tumor. ${ }^{14,23}$ En cuanto a las recidivas, se ha intentado correlacionarlas con la presencia de hallazgos de quiste óseo aneurismático en la zona tumoral sugerido por Izadpanah ${ }^{5}$ aunque ese hecho había sido negado con anterioridad en un estudio en el que solamente se halló correlación con la recidiva si la duración de los síntomas era de menos de 6 meses. ${ }^{11}$ Para tratar de evitar las recidivas se ha sugerido que tras el legrado se use una fresa a elevadas revoluciones, phenol o crioterapia. ${ }^{6,24}$ Más difícil es establecer el pronóstico en cuanto a la producción de metástasis; más aún cuando éstas pueden producirse hasta 13 e incluso 17 años tras
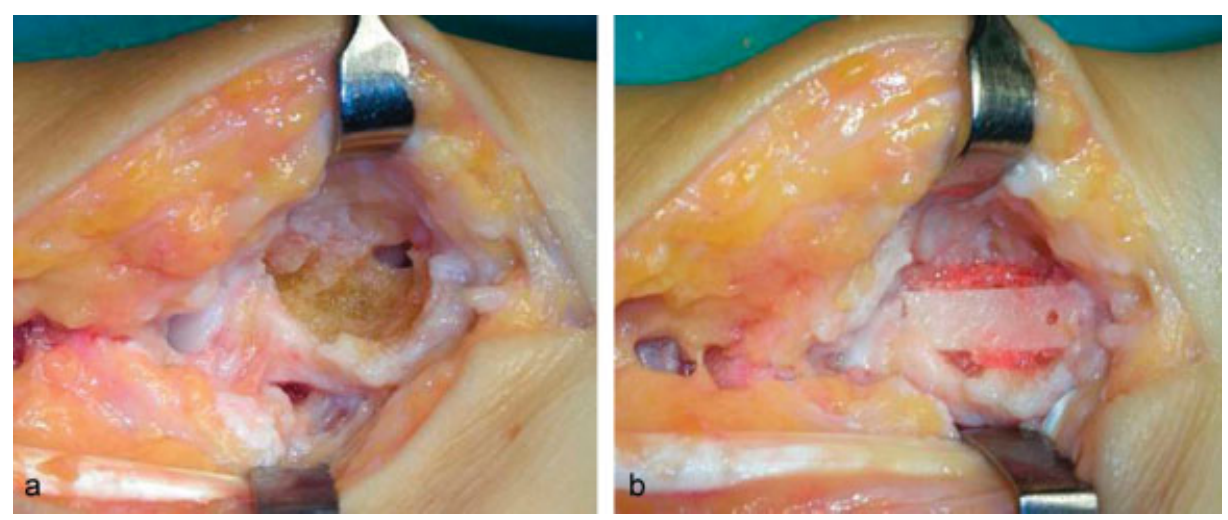

Fig. 6 (a) aspecto tras la resección de tejido tumoral. (b) colocación de un injerto esponjoso y un fragmento de cortical de la metáfisis distal del radio. 


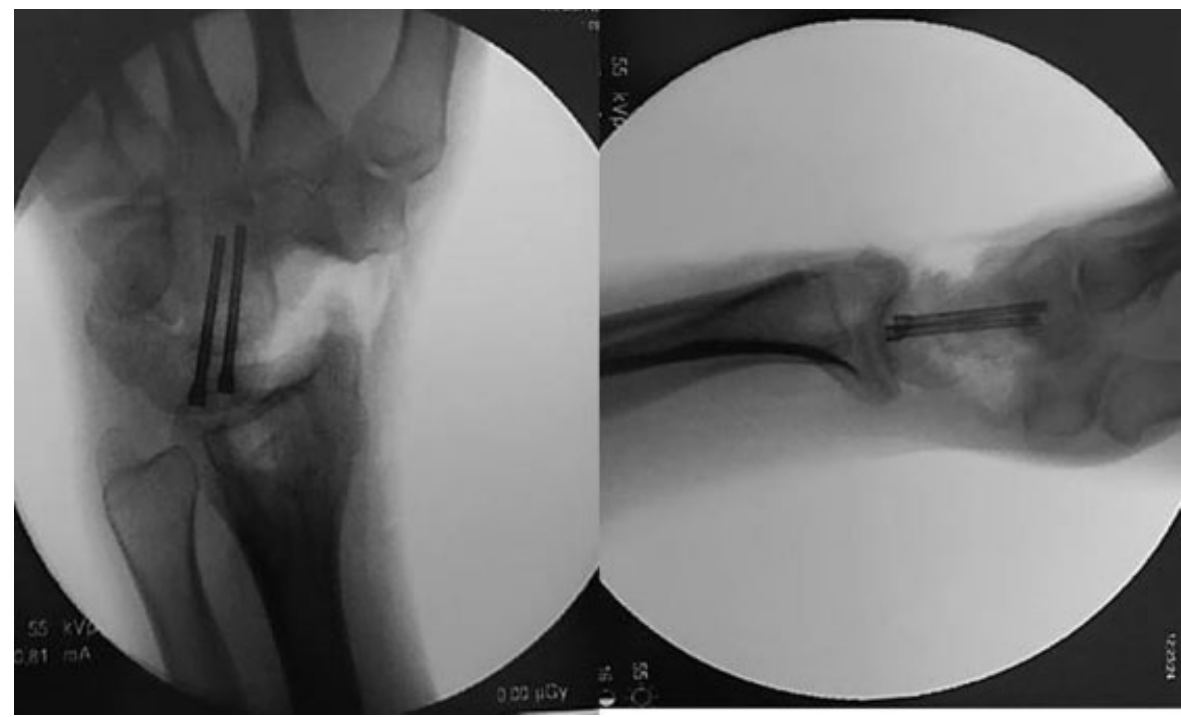

Fig. 8 Imagen intraoperatoria de artrodesis semilunar-grande con exéresis completa de escafoides.

la exéresis del tumor original. ${ }^{15,17}$ También es cierto que en muchos de los casos publicados por la aparición de metástasis, el tumor primario se hallaba en el esqueleto axial (costilla, ${ }^{17}$ pelvis, ${ }^{20,21}$ columna, ${ }^{19}$ aunque también alguno originalmente diagnosticado en astrágalo. ${ }^{18}$ Las metástasis pueden también ser diagnosticadas a la vez que el tumor óseo. ${ }^{14,18,19,25}$ La anatomía patológica de las metástasis era superponible al tumor primario con variación aneuploide. ${ }^{14,19}$

Así como en la afectación de huesos largos la recurrencia tras legrado ha sido establecida entre un $4 \%$ y $32 \%,{ }^{12}$ no se han encontrado estudios a nivel del carpo de producción de metástasis ni de frecuencia de recidivas aunque si se han publicado casos aislados de recidivas. ${ }^{4}$

Es difícil saber la cantidad de casos publicados de condroblastoma que afecta a los huesos del carpo. La serie más larga es la de $\mathrm{Wu},{ }^{4} \mathrm{y}$ menciona 19 casos añadiendo dos más que afectaban al priramidal. Sí queda claro que el hueso más frecuentemente afectado es el escafoides, con 6 casos en la serie, seguido del hueso grande. Esos dos huesos acumulan el 52\% de los casos del carpo. ${ }^{3}$ Piramidal, semilunar y ganchoso también han sido asiento de este tumor. ${ }^{3,26,27}$ Como curiosidad, el trapecio apareció como afectado por un CB en 2008 por primera vez. ${ }^{28}$ No se han reportado casos de afectación del trapezoide.

En nuestro caso, tras una primera recidiva, se consideró ya la posibilidad de realizar un tratamiento más definitivo mediante la exéresis del escafoides y una artrodesis parcial $^{5}$ pero se optó por un nuevo intento debido a la edad del paciente e intentar evitar una intervención con secuelas. La exéresis completa del hueso afecto ya se había mencionado en la literatura en el caso del ganchoso. ${ }^{27} \mathrm{El}$ informe de la segunda biopsia describiendo la lesión de condrosarcoma de bajo grado nos inclinó definitivamente a la exéresis del hueso y fijación semilunar-hueso grande. ${ }^{29}$

Por lo tanto, el pronóstico tras el tratamiento del condroblastoma benigno, debe ser cauto y prudente y no está exento de riesgo de recidiva, por lo cual algunos autores han recomendado el tratamiento con fenol, crioterapia, el uso de una fresa a altas revoluciones o incluso con radiofrecuencia. $^{30}$ Aunque raras, son posibles las metástasis que pueden ser detectadas en el mismo momento del diagnóstico de la lesión o años más tarde, pero a nivel del carpo no se conocen datos. El seguimiento de los pacientes tratados de esta lesión debe prolongarse y puede incluir estudios radiológicos ( $\mathrm{Rx}$ simple o TAC) de tórax. Tan es así, que desde 2013 ese tumor no está incluido entre los estrictamente benignos sino en un apartado de "intermediate tumors (rarely metastazing)."31

\section{Conflicto de Intereses}

Los autores declaran que no existe ningún conflicto de interés.

\section{Bibliografía}

1 Jaffe HL, Lichtenstein L. Benign chondroblastoma of bone: a reinterpretation of the so-called calcifiying of chondromatous giant cell tumor. Am J Pathol 1942;18(06):969-991

2 Unni KK, Inwards CY. Benign Chondroblastoma. En Dahlin's bone tumors: general aspects and data on 10,165 cases. 6th ed. Philadelphia: Wolters Kluwer Lippincott Williams \& Wilkins; 2010:41-48

3 Murray PM, Berger RA, Inwards CY. Primary neoplasms of the carpal bones. J Hand Surg Am 1999;24(05):1008-1013

$4 \mathrm{Wu} \mathrm{CT}$, Chen ACY, Wang CJ, Wang CW, Fu CJ, Wong YC. Chondroblastoma of the triquetrum. Pediatr Neonatol 2013;54 (04):278-280

5 Izadpanah A, Zreik RT, Shives T, Kakar S. Capitate Chondroblastoma: A Case Report and Review of the Literature. Hand (NY) 2017;12(02): NP14-NP18. Doi: 10.1177/1558944716642762

6 Davila JA, Amrami KK, Sundaram M, Adkins MC, Unni KK. Chondroblastoma of the hands and feet. Skeletal Radiol 2004; 33(10):582-587

7 Duttaluri R, Sultanpurkar GP, Raorane H, Vikram H. Malignant chondroblastoma of extraskeletal origin. Int J Appl Basic Med Res 2016;6(02):146-148. Doi: 10.4103/2229-516X.179028

8 Repáraz FJ, Garbayo J, Arrechea MA, Corchuelo C, Tejero A, Ayala H. Condroblastoma de escafoides carpiano. An Sist Sanit Navar 2008; 31(03):295-300 
9 Dahlin DC. Bone tumors. Generla aspects and data on 6.221 cases. 3rd edition. Charles C Thomas Publi. Springfield. ILL.; 1978:43

10 Vijayan S, Bhat AK, Hameed SA, Kumar B. Chondroblastoma of the scaphoid: A case report. J Cancer Res Ther 2015;11(03):669. Doi: 10.4103/0973-1482.151938

11 de Silva MV, Reid R. Chondroblastoma: varied histologic appearance, potential diagnostic pitfalls, and clinicopathologic features associated with local recurrence. Ann Diagn Pathol 2003; 7(04):205-213

12 Rhee PC, Novais EN, Shives TC, Shin AY. Chondroblastoma with secondary aneurysmal bone cyst of the hamate: case report. J Hand Surg Am 2012;37(03):538-542

13 Accadbled F, Brouchet A, Salmeron F, Darodes P, Cahuzac JP, Sales De Gauzy J. [Recurrent aggressive chondroblastoma: two cases and a review of the literature]. Rev Chir Orthop Repar Appar Mot 2001;87(07):718-723

14 Viswanathan S, Jambhekar NA, Merchant NH, Puri A, Agarwal M. Chondroblastoma of bone-not a " benign disease": clinico-pathologic observations on sixty cases. Indian J Pathol Microbiol 2004;47(02): 198-201

15 Rodgers WB, Mankin HJ. Metastatic malignant chondroblastoma. Am J Orthop 1996;25(12):846-849

16 Kyriakos M, Land VJ, Penning HL, Parker SG. Metastatic chondroblastoma. Report of a fatal case with a review of the literature on atypical, aggressive, and malignant chondroblastoma. Cancer 1985;55(08):1770-1789

17 Khalili K, White LM, Kandel RA, Wunder JS. Chondroblastoma with multiple distant soft tissue metastases. Skeletal Radiol 1997; 26(08):493-496

18 Jambhekar NA, Desai PB, Chitale DA, Patil P, Arya S. Benign metastasizing chondroblastoma: a case report. Cancer 1998;82 (04):675-678

19 Sohn SH, Koh SA, Kim DG, et al. A case of spine origin chondroblastoma metastasis to lung. Cancer Res Treat 2009;41 (04):241-244. Doi: 10.4143/crt.2009.41.4.241
20 Tamura M, Oda M, Matsumoto I, Sawada-Kitamura S, Watanabe G. Chondroblastoma with pulmonary metastasis in a patient presenting with spontaneous bilateral pneumothorax: Report of a case. Surg Today 2011;41(10):1439-1441. Doi: 10.1007/ s00595-010-4469-8

21 Lin PP, Thenappan A, Deavers MT, Lewis VO, Yasko AW. Treatment and prognosis of chondroblastoma. Clin Orthop Relat Res 2005; 438(438):103-109

22 Gómez A, Mora-Ríos FG, Cortés J. Condroblastoma primario agresivo, reporte de caso y revisión de la literatura. Revista Mexicana de Ortopedia Pediatrica 2008;10:5-9

23 Pösl M, Werner M, Amling M, Ritzel H, Delling G. Malignant transformation of chondroblastoma. Histopathology 1996;29 (05):477-480

24 Fang Z, Chen M. Chondroblastoma associated with aneurysmal cyst of the navicular bone: a case report. World J Surg Oncol 2013; 11:50. Doi: 10.1186/1477-7819-11-50

25 Green P, Whittaker RP. Benign chondroblastoma. Case report with pulmonary metastasis. J Bone Joint Surg Am 1975;57(03):418-420

26 Tountas CP, Cobb SW. Chondroblastoma of the lunate: a case report. J Hand Surg Am 1992;17(03):466-467

27 Daly KE, Chow JW, Vickers RH. Excision of the hamate for an unusual hand tumour. J Hand Surg [Br] 1993;18(05):606-608

28 Konishi E, Okubo T, Itoi M, Katsumi Y, Murata H, Yanagisawa A. Chondroblastoma of trapezium with metacarpal involvement. Orthopedics 2008;31(04):395

29 Kirschenbaum D, Schneider LH, Kirkpatrick WH, Adams DC, Cody RP. Scaphoid excision and capitolunate arthrodesis for radioscaphoid arthritis. J Hand Surg Am 1993;18(05):780-785

30 Xie C, Jeys L, James SL. Radiofrequency ablation of chondroblastoma: long-term clinical and imaging outcomes. Eur Radiol 2015;25(04): 1127-1134. Doi: 10.1007/s00330-014-3506-1

31 Chen W, Difrancesco LM. Chondroblastoma: An Update. Arch Pathol Lab Med 2017;141(06):867-871. Doi: 10.5858/arpa.20160281-RS 